The chapters on foetal maturity and foetal abnormalities will be of value to radiologist and obstetrician alike. The detailed studies of radiological maturity made in the Manchester school are well known and are beautifully presented here and the value of ultrasound in foetal cephalometry described. Obstetricians will particularly value the chapter on antenatal diagnosis of abnormalities, as the necessity increases to plan the approach to their management from the moment of birth.

The various methods of placental localization are compared and the general superiority of ultrasound demonstrated, plain radiography being second best. On pelvimetry, general principles and some pitfalls are clarified and some recent work presented; the technique preferred by $\mathrm{Dr}$ Russell is lucidly described, though it is not to be found in all X-ray departments. The final chapter on radiography of the mother clarifies several controversial issues, particularly antenatal chest films.

This book is clearly required reading for all radiologists in training and those already in practice will enjoy it with benefit. Obstetricians will chiefly gain from the chapters on radiation hazards and foetal maturity and abnormality but there is much food for thought throughout. All in all, this is an up to date authoritative guide to clinical radiological practice in obstetrics. Even at $£ 4.95$ in paperback it is a very good buy.

\section{Injuries of the Spinal Cord-The Management of Paraplegia and Tetraplegia}

By Neville G. Sutton. London: Butterworths, 1973. $£ 5.50$.

The author, a former Professor of Surgery in the University of Queensland, became Director of the Spinal Injuries Unit in Brisbane on his retirement and has now written this admirable, compact yet comprehensive, and very readable book. It begins with a short historical review of the development of spinal units, points out the desirable features of such units and reviews the future outlook on a global geographical scale. There follow chapters on the anatomy of the vertebral column and spinal cord, including the vascular supply, and on the applied surgical anatomy of the spinal cord and autonomic nervous system. After chapters on vertebral column injury, the mechanism of injury to the cord and the epidemiology of cord injury, the remainder of the book (more than half) is concerned with treatment. Treatment begins at the site of the accident and the section on first aid is both practical and topical. This is followed by individual chapters on early hospital treatment of paraplegia and tetraplegia, later hospital care, management of autonomic system dysfunction and detailed consideration of special problems such as respiratory complications, urinary tract infections, pressure sores, heterotopic calcification. The book ends with chapters on rehabilitation and after-care in which important subjects such as the patient's sexual problems are dealt with in realistic terms. There is also a wealth of practical advice on such topics as architectural modifications to accommodate the disabled, vocational homes, and life expectancy.

The book is well illustrated with photographs, diagrams, and radiographs and is beautifully produced. Although interest in this subject has grown immeasurably in recent years and a specialist journal (Paraplegia) has existed since 1965 there has been a need for an up-to-date synthesis of current views on the management of spinal-injured patients. This book will appeal to neurologists, surgeons, and to all those concerned with rehabilitation of the disabled. It can be warmly recommended.

\section{The ECG Made Easy}

By J. R. Hampton. Pp. 68, illustrated. Edinburgh and London: Churchill Livingstone, 1973. £0.80.

Bravely, the author states two principles: the ECG is easy to understand, and most abnormalities of the ECG are amenable to reason. Regrettably, he is the victim of his own lack of appreciation of electrocardiographic principles. The idea of an extremely brief and simple introduction that will provide practical knowledge is an attractive one, but this requires the author to have a sound understanding of his subject. This is sadly lacking. Errors abound, instanced by the following few examples. On p. 20, the illustration for Mobitz Type II block actually represents a blocked atrial extrasystole; on p. 27 the escape rhythm is junctional and not atrial: he fails to appreciate that the varying PR interval is due to atrioventricular dissociation and not activation of different parts of the atrium (as can be seen from his illustration, the P-P intervals are constant); on p. 28 the illustration indicates transient sinoatrial block; on p. 32 the significance of the $P$ wave following the escape beat (it is retrograde) is not appreciated; on p. 36 the discussion of the mechanism of junctional tachycardia is wrong; on p. 38 the illustration of ventricular tachycardia looks much more like atrial fibrillation with bundle branch block. There are numerous other examples that could be quoted, but a few different aspects also arise. In none of the examples in Chapter 2 and in only some in Chapter 3, are the leads indicated; the tracing on p. 37 indicates a machine fault; and the table correlating ECG appearances with rhythm and treatment is sketchy, in places inaccurate, and unsuitable as a guide for the sort of person for whom the book is intended. Regrettably, this book is likely to cause confusion rather than to advance understanding of an important topic.

\section{Ophthalmic Syndromes}

By H. V. Nema. Pp. 355. London: Butterworths, 1973. $£ 4.95$.

There are 354 syndromes described in this book. They are listed alphabetically, usually using the eponymous title but otherwise a descriptive title. Each is described in the same way. First the synonyms for the condition are given, then its main clinical features defined. The aetiology and heredity are given, when known, and then the ocular and extra-ocular features are described in more detail. Each syndrome is referenced and there is often a reference to the original description. There follow two appendices. The first classifies the syndromes according to ocular signs and the second according to extra-ocular signs.

There are those who decry the use of eponyms but this book demonstrates their use, if only as a convenient shorthand to replace a cumbersome description. Furthermore, eponyms are part of the history of medicine and commemorate (albeit often inaccurately) the efforts of our predecessors and colleagues. There is a curious anomaly in that the eponyms are sometimes relegated to the synonym section while a descriptive title is used. Some of the syndromes appear with little ophthalmological justification; the supraorbital pain of Costen's Syndrome seems little reason for its inclusion.

Many ophthalmic conditions are rare and many of these syndromes are very rare. An awareness of the existence of these syndromes may help place many a puzzling clinical finding. A great deal of work has gone into this book and, as the foreword states, it will be a most useful source of information. The most likely reader will be the candidate for the higher ophthalmic examinations (or the examiner). 